\title{
Design and Implementation of Mobile Electronic Nautical Chart Information System Based on IOS
}

\author{
Zijie Jiang ${ }^{1}$, Zhongliang Cai ${ }^{*}$, Hui Zhou ${ }^{1}$, Nian Hui ${ }^{2}$ \\ ${ }^{1}$ School of Resource and Environmental Sciences, Wuhan University, Wuhan 430079, China \\ 2Department of Navigation Data, Wuhan Kotei Informatics Co., Ltd., Wuhan 430079, China \\ * Corresponding author. Email: zlcai@whu.edu.cn \\ Manuscript submitted March 8, 2015; accepted June 31, 2015. \\ doi: 10.17706/jsw.10.7.835-841
}

\begin{abstract}
With the rapid development of nautical surveying and mapping and the growing of the mobile platform products, there is a growing demand for mobile electronic nautical chart information system. Traditional desktop electronic chart information system has played an important role in the field of hydrographic surveying and mapping. But it is very inconvenient to carry it offshore for maritime staff. We have developed the mobile electronic nautical chart information system based on IOS after deeply digging the demand of maritime staff for a mobile system. It realizes some traditional functions like the desktop system such as parsing and rendering of vector nautical chart file, integrated display of various kinds of map layers, data update and sharing inside the system and so on. Besides that, it also realizes some novel functions taking advantage of the characteristics of the mobile devices, such as trajectory recording and navigation, etc. This system is on trial in the relevant operational departments and reaches the level of operational application.
\end{abstract}

Key words: Nautical chart, information system, IOS, Objective-C++.

\section{Introduction}

With the development of space science, information science and computer science, a lot of changes have been made in the field of nautical surveying and mapping. The scale and application scope is getting larger and larger. And it's also developing from simple surveying and mapping work to efficient production and management of surveying and mapping data and application services [1].

In recent years, the rapid development of mobile Internet has brought many new development opportunities to all walks of life. It changes the way people get information, which in turn changes the development of all kinds of information system. And nautical chart information system is not exceptional either. How to develop more special functions on the basis of its typical functions according to the characteristics of mobile environment on nautical chart information system is the subject of present research.

At present, most nautical chart information systems are running on the desktop. And there are only a few mobile nautical chart information systems, which only have some basic functions, such as display of raster chart, publishing and browsing of basic information, etc [1]-[3]. However what users really need is a mobile nautical chart information system which has most of the practical functions provided by traditional desktop nautical chart information system [4]. In order to meet this demand, we have developed a mobile nautical chart geographic information system based on IOS. 
Therefore, the remainder of this article is organized as follows. We start in Section 2 with discussion of the overall application analysis. We continue in Section 3 with an overall system architecture and a detailed implementation of the system. We end in Section 4 with discussion and an outlook for a future work.

\section{Application Analysis}

This paper mainly aims to research on the integrated display of various types of electronic chart layers, the data synchronization problem between the server and mobile devices and how to deeply utilize the characteristics of the mobile platform to develop unique functions.

\subsection{Development Mode}

ArcGIS has always been the widely used GIS software. The great functions such as map display, data organization and geographic analysis provided by its desktop version hasbrought much convenience to GIS workers. And it provides development tools throughout the various development platforms, including IOS, which is one of the most popular mobile operating systems [3]. ArcGIS Runtime SDK for IOS is such one development framework which provides the most basic related functions of a geographic information system under IOS platform [5]. In order to be able to concentrate on the top application layer, we chose ArcGIS Runtime SDK for IOS as our underlying framework instead of developing from scratch. It can easily implement many basic functions such as raster map display, map service access, map layer management, coordinate system setting, measurement on the map and so on. It laid a solid foundation for the subsequent advanced functions as well.

However, ArcGIS Runtime SDK for IOS does not support parsing and rendering of the vector format of electronic nautical chart, namely 000 file which conforms to international electronic nautical chart S57 standard. After comparison of different development modes, we finally choose a mixed mode for parsing and rendering of vector electronic nautical chart, which means that we use standard $\mathrm{C}++$ to parse it and IOS specific programming language Objective-C to render it. The parsing module is implemented using standard C++ in order to be run cross-platform. So if we want to develop our nautical chart information system under Android or other systems later on, the parsing module can be used directly without modification. And the rendering module is implemented using Objective-C considering that rendering mechanisms are so different across different platforms. So it is not developed as cross-platform module. Besides that, rendering process can also speed up taking the advantage of the self-owned rendering engine of different platforms.

\subsection{Research Contents}

\subsubsection{Preprocessing and packaging of raw data}

A variety of multisource raw data must be preprocessed, packaged and stored into database before getting into the mobile nautical chart information system. After this kind of process, the data will have consistent contents and format and will be easily recognized and used by mobile nautical chart information system. This process includes the classification of the data, the transformation of coordinate system, the unification of data accuracy, putting into database and some other special operations. For example, raster image processing includes the original image geo-referencing, raster data packagingin ArcGIS Desktop, and the final putting into database operations.

\subsubsection{Integrated display of different kinds of map layers}

As an integrated electronic nautical chart information system, it should support integrated display of various types of map layers. This includes electronic chart, tiled package of electronic chart, tiled package of paper chart, satellite image, surveying and mapping data of other formats as well as online map services and so on. Every kind of map layers has its own unique supported operations. So the system should provide 
different containers to hold these different map layers. These different layers should coexist harmfully and the transition between them should be seamless. At the same time, their symbolization should also be uniform.

\subsubsection{Data synchronization between servers and clients}

Since the nautical environment is changing all the time, our electronic nautical information system must keep its data really real-time to provide reliable guidance to marine work. From the perspective of this view, data update is a major problem that we must face in our nautical information system. We have adopted the widely used C/S model that is very common in IOS network programming [9]. And the client here is our mobile nautical chart information system. Aiming at different kinds of data, we have set up different data update strategy. This includes data that needs frequent update such as navigation mark information, and data that needs not so frequent update such as paper chart. Both of them have their corresponding update strategies.

\subsubsection{Utilization of characteristics of mobile devices}

Compared to traditional desktop nautical chart information system, the mobile one has more advantages. Not only does it have higher portability, but also more novel functionscan be developed taking advantage of the mobile devices. One outstanding example is that some specific functions can be developed using the various sensors of the mobile devices. For example, GPS receiver can be used to get the device location information. When combined with the nautical chart and other navigation information, ships can be tracked and navigated by our nautical chart information system.

\section{System Design and Implementation}

\subsection{System Overall Design}

Our mobile nautical chart information system can be generally divided into four modules, which are data organization, map display application features and user management \& system settings. And application features include many small functions. The following overall architecture diagram has showed this:

\subsection{Detailed Implementation}

\subsubsection{Vector electronic chart rendering}

Since the system is built on the top of ArcGIS Runtime SDK, all the map layers must reside in the map view control named AGSMapView provided by ArcGIS. Of all the map layers provided by ArcGIS, AGSDynamicLayer is capable of providing custom vector rendering. So our vector electronic nautical chart rendering module is implemented by inheriting from this AGSDynamicLayer. In order to make the vector electronic chart parsing module independent from platforms and make it easier to port the whole nautical chart information system across different platforms, the platform-independent programming language standard $\mathrm{C}++$ is adopted as our programming language for the vector nautical chart parsing library. The IOS developing IDE Xcode provides very high support for standard $\mathrm{C}++$ though its suggested programming language is Objective-C[6]. It can call the function inside a standard C++ encapsulated library, whether it is static library or dynamic library. It also support mixed coding of Objective-C and standard $\mathrm{C}++$ [7]. After comparing these two modes, we decided to encapsulate the vector nautical chart parsing module into a static library called framework to maintain the modules' independence from each other. Parsing module can be also divided into two parts, one is vector nautical chart file parsing, which corresponds to nautical chart transportation standard S57, the other one is the symbol library parsing, which corresponds to nautical chart display standard S52.

Considering that different platforms have more support and higher performance for their own rendering engines and it's also much more convenient to use their own rendering engines[8], we'll use the IOS 
rendering engine Core Graphics to render the vector nautical chart.

In general, the nautical chart files and symbol library files are parsed into nautical chart object ENCProject that resides in memory using the parsing module written by standard $\mathrm{C}++$. After that the rendering module written by Objective-C is responsible to render ENCProject to raster image. Then, the raster image is used to fill the ENCLayer that inherits from AGSDynamicLayer. At last, the ENCLayer gets loaded into AGSMapView together with other kinds of map layers to form a complete nautical chart view.

The whole framework is shown in Fig. 1 below:

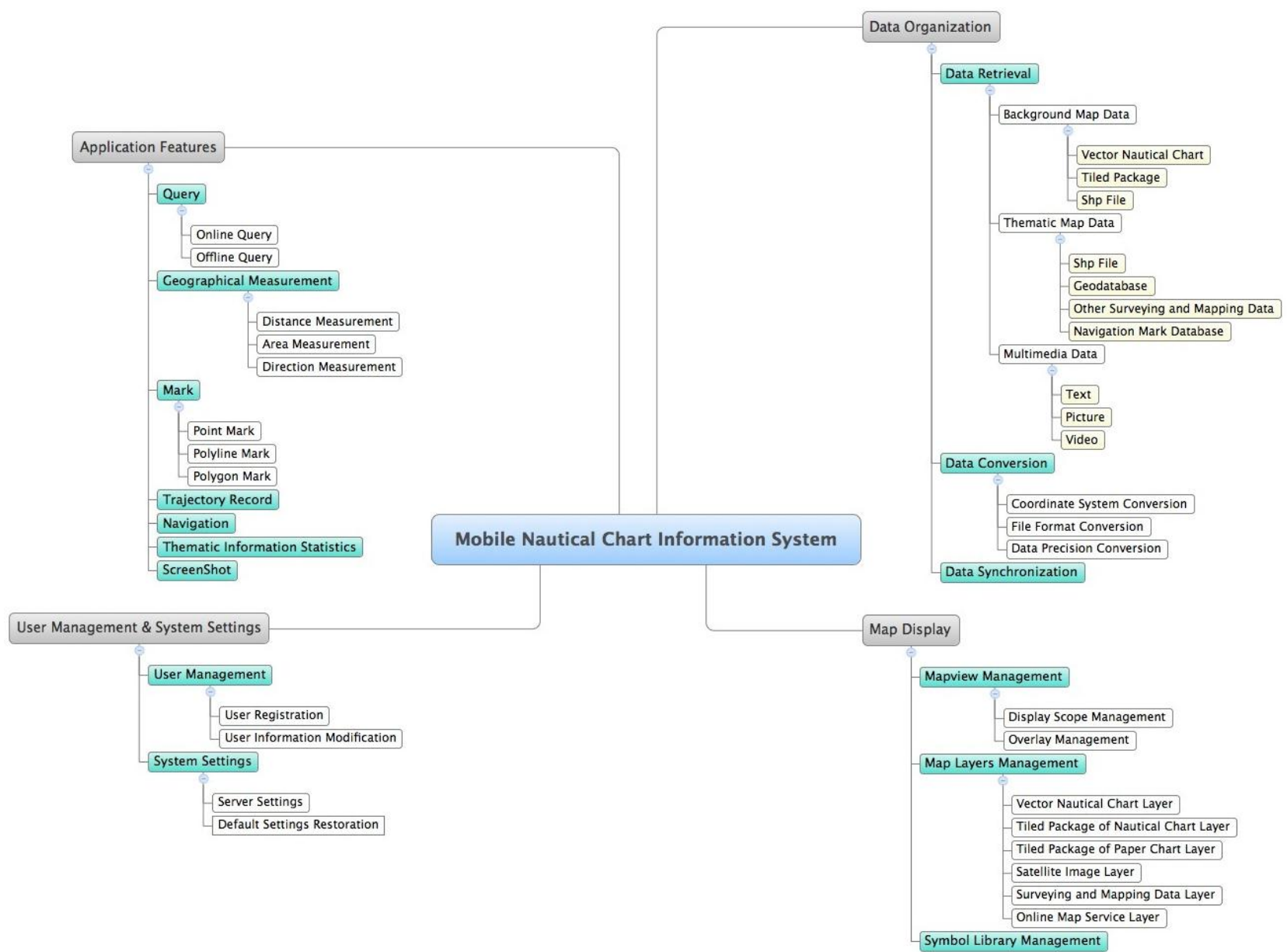

\subsubsection{Self-adaptive display}

Fig. 1. Overall design.

The system contains a variety of kinds of map layers. And each map layer has its own various display styles. So the map interface will become a messif there is not a set of reasonable and effective display control mechanism to manage the information to display. It's very common in traditional desktop nautical chart information system to provide a large number of configuration options to allow the user to adjust the map display. But in mobile nautical chart information system, self-adaptive display is more widely used. This is mainly attributed to the screen size and usage habits of mobile devices. In the mobile nautical chart information system, we try our best to infer users' potential demand and preference from the basic information that we have got already to adjust the map display.

The information taken into consideration for self-adaptive display includes the basic information of the user, the current environmental information, such as time, weather, temperature, etc., the current location information, and users' operation records. The display adjustment also covers many aspects, such as the switchover between traditional and simplified symbols of vector nautical chart, the switchover between day and night modes, the automatic switchover among different kinds of map layers, map contents 
adjustment depending on different scales and so on. The combination of these adjustments will eventually form a complete set of adaptive display mechanism and provide a stable and efficient display basis for the nautical chart information system (see Fig. 2).

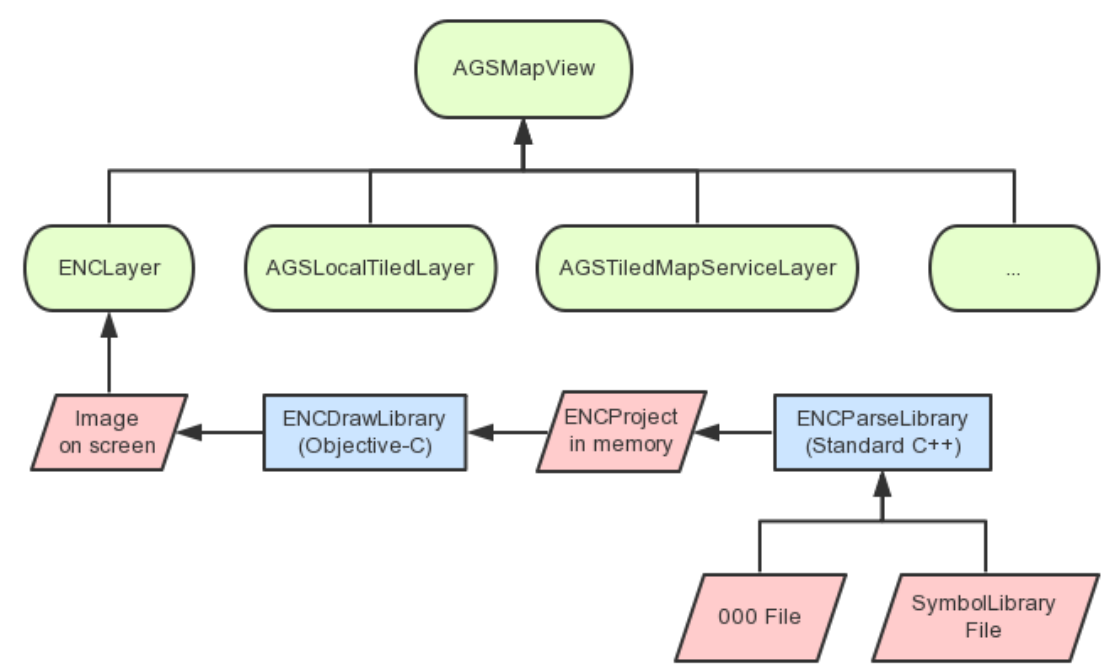

a. Flow chart.

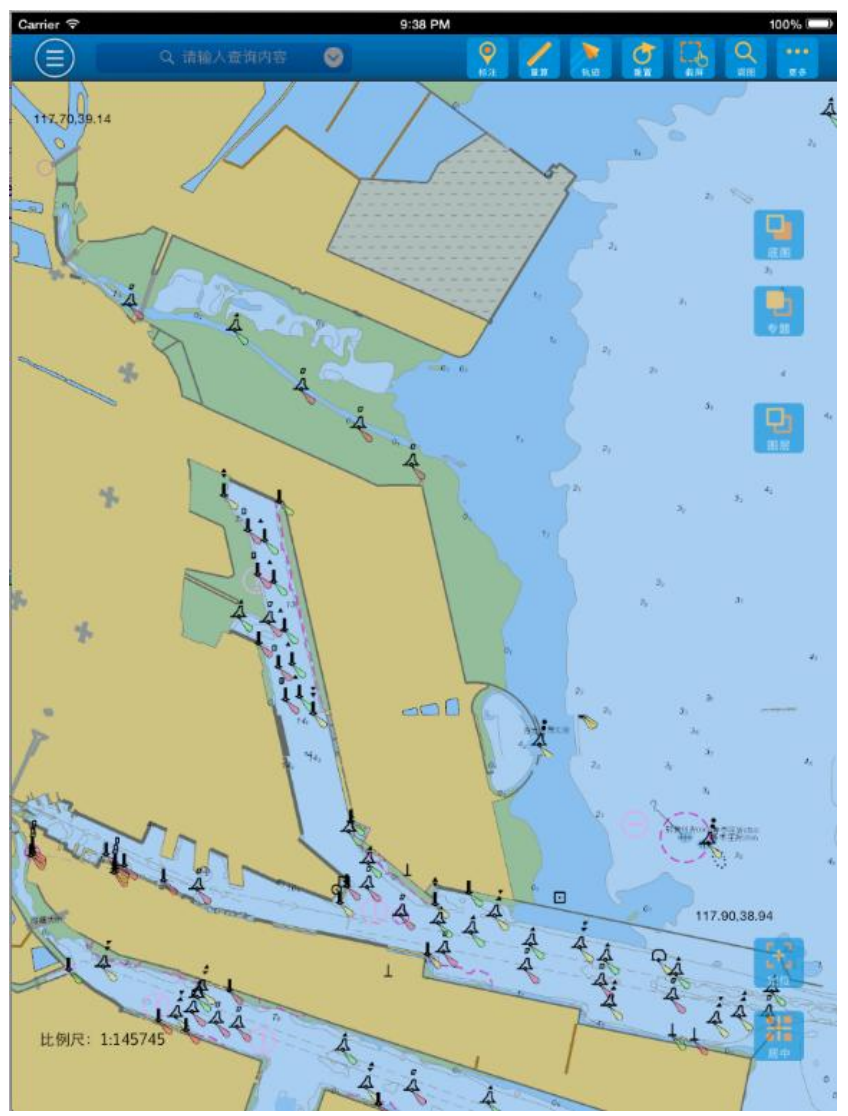

b. rendering.

Fig. 2. Vector nautical chart rendering.

\subsubsection{Data synchronization}

In the system, different data have different update strategies considering their types and update frequencies. Some data that need not so frequent update such as vector nautical chart and tiled package of paper chart can be updated through downloading newer data from server to override the outdated data of the mobile device. Local update of vector nautical chart can also be achieved by append vector nautical 
chart correction file 001,002 , and so on to vector nautical chart 000 file. And some other data that need very frequent update such as navigation mark information can be updated by accessing the remote database for real-time display. Both of the two strategies involved the data transportation problem between mobile devices and the remote server. Since mobile devices cannot access the database service of the remote server directly, there must be an intermediate web service layer between mobile devices and remote server database service to transfer the data. In brief, the intermediate web service layer is a proxy. It listens to mobile devices' request, comprehends it and then sends its own data request to the remote database server. After the remote database server sends back its data response, the intermediate web service layer receives it, comprehends it and then sends its own data response to mobile devices. Nautical chart file upload and download process has adopted the similar pattern. We have adopted the widely used web service library CFNetwork [9] to handle web service related operation in IOS. And WCF web service is deployed using Visual Studio in Windows to act as the intermediate web service layer (see Fig. 3).

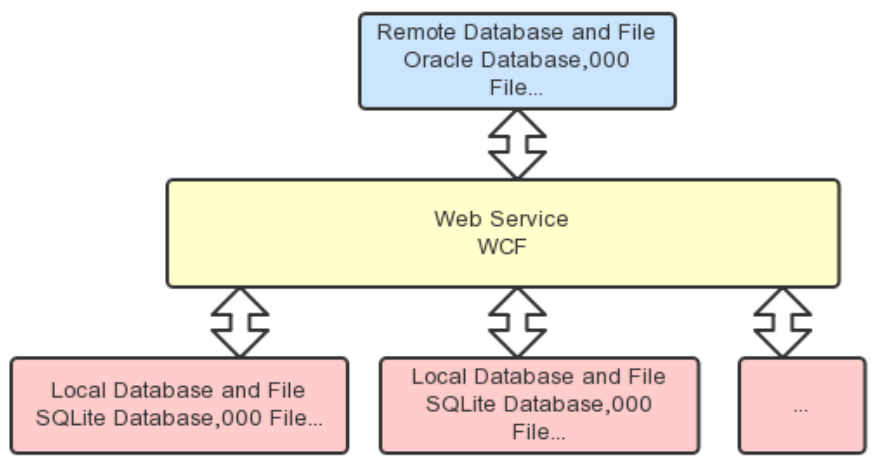

Fig. 3. Data synchronization.

\subsubsection{Utilization of location information}

Ships carrying our mobile nautical chart information system can be positioned using GPS, WI-FI or mobile network. We have utilized the IOS system provided library Core Location to deal with the location information. Based on that, ship sailing trajectory recording function can also be realized. In other words, the location of the ship can be continuously recorded during sailing and stored in the end. The trajectory information can be uploaded to the server finally. So it can be shared between all the ships carrying our electronic nautical chart information system. In addition, the realization of the positioning function has brought the possibility of navigation. Real-time water depth information and other sailing information can be accessed through the remote database server during sailing. This can make sailing more safe and efficient. And these functions are novel functions that the traditional desktop nautical chart information system can't realize.

\section{Conclusion}

The mobile electronic nautical chart information system built in this paper is based on ArcGIS Runtime SDK for IOS in general. It adopts a mixed mode to parse and render vector nautical chart file. In the system, various kinds of map layers are supported taking advantage of ArcGIS SDK abilities. An intermediate web service layer is introduced to solve the data transmission problem between server and mobile devices. And location data can also be utilized for trajectory recording and navigation. The system in this paper is on trial, and it has improved the nautical surveying and mapping work efficiency and changed the way they work. The mixed mode of parsing and rendering vector nautical chart file and the introduction of the intermediate web service layer can provide some technical help and advices for other similar information system. 


\section{Acknowledgment}

The authors thank Ming Li and Meng He for their assistance and appreciate the reviewers' valuable comments.

\section{References}

[1] Fowler, C., \& Treml, E. Building a marine cadastral information system for the United States-A case study. Computers, Environment and Urban Systems, 25(4), 493-507.

[2] Ward R, Roberts C, \& Furness R. (2000). Electronic chart display and information systems (ECDIS): State-of-the-art in nautical charting. Marine and Coastal Geographical Information Systems.

[3] Ni, Y, Chen, B, Ye, P., et al. (2014). A framework for IOS application development. Journal of Software, 9(2), 398-403.

[4] Marine and Coastal Geographical Information Systems. CRC Press.

[5] Goyal, D., \& Dostal, M. Building Applications with ArcGIS Runtime SDK for iOS-Part I.

[6] Conway, J., \& Hillegass, A. (2012). IOS programming: The Big Nerd Ranch Guide. Addison-Wesley Professional.

[7] Cable, L. P. G. (1999). Method and apparatus for mixing objective-C and C++ objects: U.S. Patent.

[8] Munshi, A., Ginsburg, D., \& Shreiner, D. (2008). OpenGL ES 2.0 programming guide. Pearson Education.

[9] Cox, J., Jones, N., \& Szumski, J. (2012). Professional IOS Network Programming: Connecting the Enterprise to the IPhone and IPad. John Wiley \& Sons, 2012.

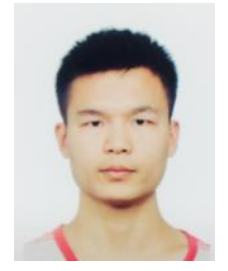

Zijie Jiang received his bachelor degree in surveying and mapping engineering from Wuhan University in 2013. He is currently pursuing the M.S. degree with the major of cartographic and geographic information system in Wuhan University. His research interests include digital map making and GIS software developing method.

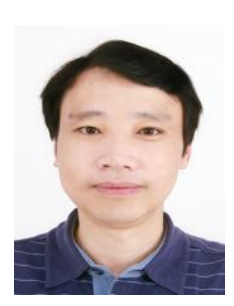

Zhongliang Cai was born in Shandong, China. He received his B.E. and M.S. degree in map making in Wuhan Surveying and Mapping Technology University, China, in 1996 and 1999, respectively. And he received the $\mathrm{PhD}$ degree in map making and geographic information engineering, School of Resource and Environmental Sciences, Wuhan University, China, in 2004. Now he works as a professor in School of Resource and Environmental Sciences in WHU and his research interests include digital map theory and method, geographic information service and GIS software developing method.

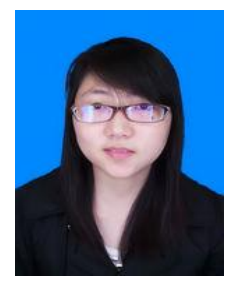

Hui Zhou has been studying at Wuhan University since 2009. She received her BS degree in geographic sciences from Wuhan University in 2013 and she is pursuing the M.S. degree in cartographic and geographic information system. Her research interests mainly include electronic map designing and developing method.

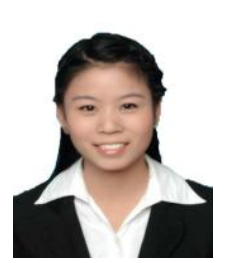

Nian Hui received her bachelor degree in urban planning from Wuhan University in 2012 and M.S. degree in surveying and mapping engineering from Wuhan University in 2014, respectively. She now works at Wuhan Kotei Informatics Co., Ltd. as a navigation system programmer in Wuhan, China. Her research focuses on navigation data processing and GIS software developing method. 